\title{
Progressive reduction of heart rate variability with eventual sudden death in two patients
}

\author{
M Nakagawa, T Saikawa, $M$ Ito
}

\begin{abstract}
In two patients with old myocardial infarction and congestive heart failure there was a progressive reduction of heart rate variability and eventual sudden death. The two patients had had three $24 \mathrm{~h}$ electrocardiogram recordings within two years of death. The power of two spectral bands was calculated- $-0 \cdot 04-0 \cdot 15$ $\mathrm{Hz}$, low frequency power, and 0.15-0.40 $\mathrm{Hz}$, high frequency power. The mean low and high frequency powers over the $24 \mathrm{~h}$ recording progressively decreased whereas the frequency of ventricular arrhythmias showed no consistent changes in either patient. The circadian variation in hourly low and high frequency powers in the last $24 \mathrm{~h}$ electrocardiogram recordings was much reduced. These results suggest that sequential measurements of heart rate variability may be useful in predicting sudden death.
\end{abstract}

(Br Heart $\mathcal{F} 1994 ; 71: 87-88)$

A reduction in heart rate variability has been linked to a high risk of sudden death in patients with coronary artery disease. ${ }^{1-3}$ However, to our knowledge, the time course of changes in heart rate variability during long-term follow up of such patients has not been examined before. We describe two patients with old myocardial infarction and congestive heart failure in whom there was a progressive reduction of heart rate variability and eventual sudden death.

\section{Case reports}

Case 1, a 58-year-old man, died suddenly from ventricular fibrillation in hospital. Case 2, a 56-year-old man, had been followed in our outpatient clinic. He died suddenly at home immediately after severe chest pain

Frequency of ventricular arrhythmias, heart rate variability, and ejection fraction in cases 1 and 2

\begin{tabular}{|c|c|c|c|c|c|c|c|}
\hline & \multicolumn{3}{|c|}{ Case 1: Months before death } & & \multicolumn{3}{|c|}{ Case 2: Months before death } \\
\hline & 16 & 6 & 0.5 & & 20 & 14 & 3 \\
\hline VPCs/day & 1218 & 167 & 1420 & . & 1430 & 376 & 55 \\
\hline VT/day & 1210 & 0 & 0 & & 0 & 1 & 0 \\
\hline $\mathrm{mLF}$ & 7 & 9 & 4 & & 15 & 11 & 5 \\
\hline $\mathrm{mHF}$ & 9 & 10 & 3 & & 10 & 7 & 4 \\
\hline $\mathrm{EF}(\%)$ & 49 & 41 & 40 & & 46 & 45 & 36 \\
\hline
\end{tabular}

VPC, ventricular premature contraction; VT, ventricular tachycardia; $\mathrm{mLF}$, mean low frequency power for $24 \mathrm{~h}$; mHF, mean high frequency power for $24 \mathrm{~h}$; EF, left ventricular ejection fraction measured by echocardiography. developed. An electrocardiogram at the time of his death was not available. Both patients had had three 24 hour electrocardiograms recorded in the two years before they died. In one (case 1) the recordings were made 16,6 , and 0.5 months before death and in the other (case 2) 20, 14, and 3 months before death. The $24 \mathrm{~h}$ electrocardiogram recordings were analysed with a Marquette 8000 scanner. The power spectrum of the RR intervals was computed by a fast Fourier transform and expressed as the square root of the areas under the power spectrum. We calculated the power of two spectral bands- $0.04-0.15 \mathrm{~Hz}$ (low frequency power) and $0 \cdot 15-0.40 \mathrm{~Hz}$ (high frequency power). The low and high frequency components during consecutive two minute periods were averaged for every consecutive hour (hourly low and high frequency powers) and for $24 \mathrm{~h}$ (mean low and high frequency powers). The frequency of ventricular arrhythmias during the 24 hours was also determined. The left ventricular ejection fraction was measured by echocardiography.

The table shows the time course of the changes in the measured variables. In both patients the mean low and high frequency powers and ejection fraction tended to decrease progressively as the time of sudden death approached. The frequency of ventricular arrhythmias showed no consistent changes, however. The figure shows the circadian variation of the hourly low and high frequency powers of the first and the third (last) $24 \mathrm{~h}$ electrocardiograms in the two patients. Healthy individuals typically show a predominance of low frequency power during the day and of high frequency power at night. ${ }^{4}$ In the first recording (open circles) the pattern of circadian variation in case 1 was abnormal for low frequency power (predominance only during the early morning) (A) but almost normal for high frequency power (B) whereas case 2 showed an almost normal low frequency power pattern (C) but an abnormal high frequency power pattern (predominance during the day) (D). In the last recordings (closed circles) both low and high frequency powers decreased markedly and there was little circadian variability in either patient.

\section{Discussion}

Others have described an association between a decreased heart rate variability and an increased risk of sudden death in patients with coronary artery disease. ${ }^{1-3}$ These studies were based on one measurement of heart rate 
Circadian variation in hourly low (LF) and high frequency powers (HF) in case $1(A, B)$ and case 2 $(C, D)$ during first $(O)$ and third (O) $24 \mathrm{~h}$ ECGs. The amplitude and ECGs. The amplitude and
circadian variation in $L F$ circadian variation in $L F$ ECGs were much reduced.
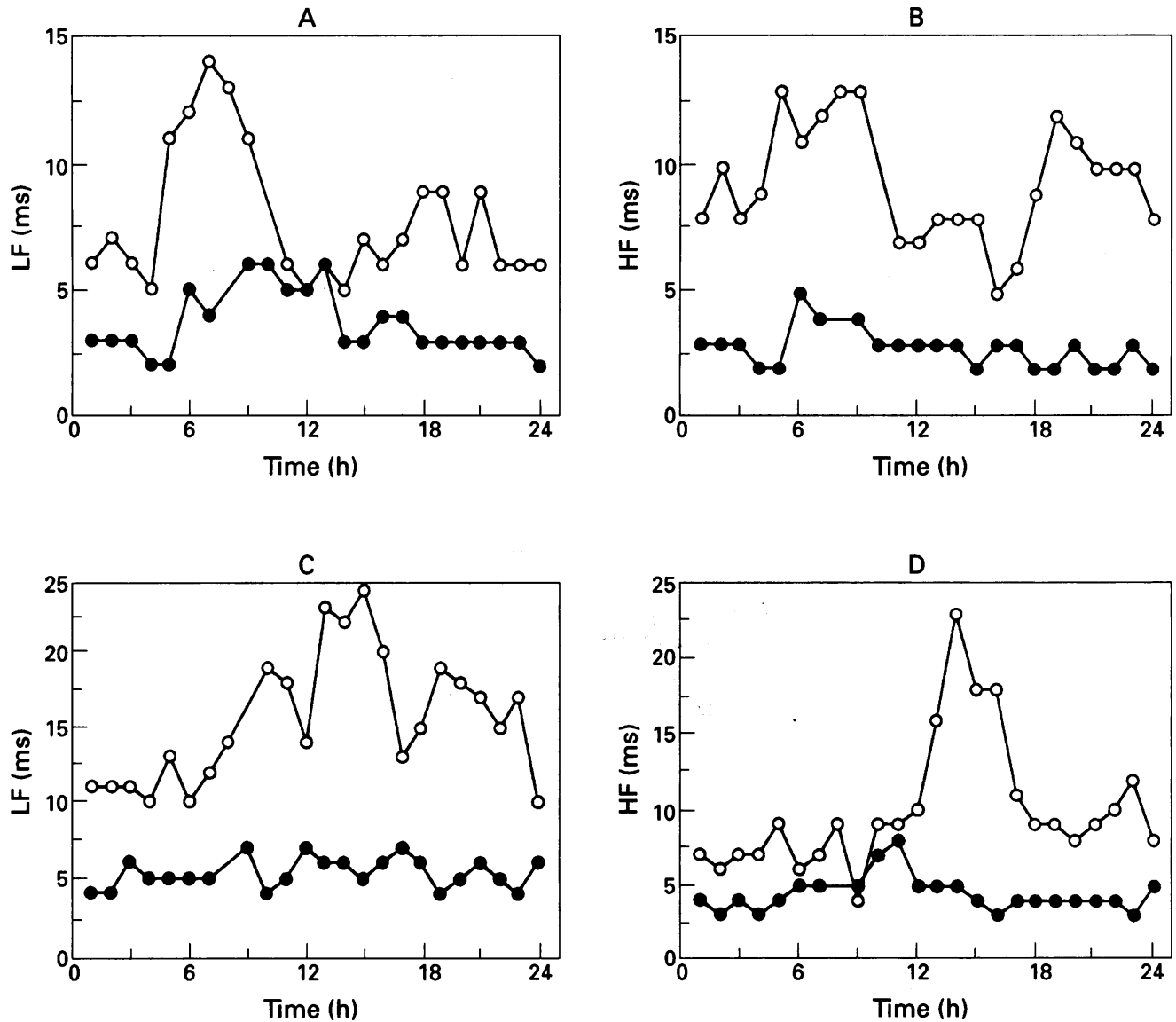

variability. ${ }^{1-3}$ We showed a progressive reduction of heart rate variability in our patients as they approached sudden death. This suggests that sequential measurements of heart rate variability may be useful in predicting sudden death. More patients need to be studied to confirm this association.

1 Martin GJ, Magid NM, Myers G, Barnett PS, Schaad JW, Weiss JS, Lesch $M$, Singer DH. Heart rate variability and sudden death secondary to coronary artery disease

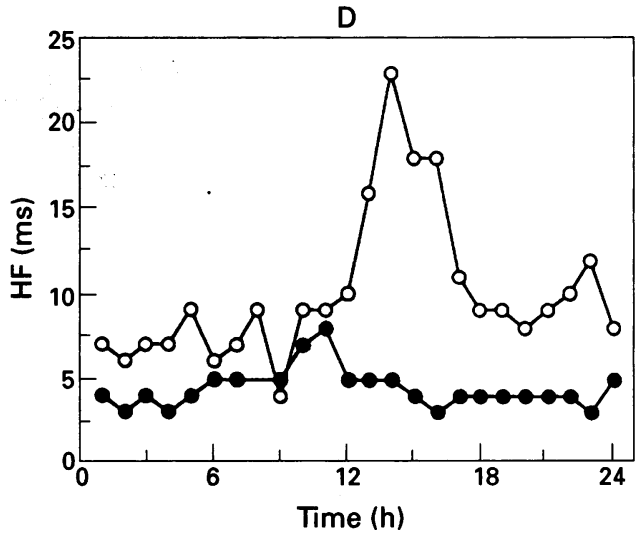

during ambulatory electrocardiographic monitoring. $A m$ 7 Cardiol 1987;60:86-9.

2 Odemuyiwa O, Malik M, Farrell T, Bashir Y, Staunton A Poloniecki J, Camm AJ Multifactorial prediction of arrhythmic events after myocardial infarction. Combination of heart rate variability and left ventricular ejection fraction with other variables. PACE 1991; 14:1986-91.

3 Bigger JT, Fleiss JL, Steinman RC, Rolnitzky LM, Kleige RE, Rotman JN. Frequency domain measures of heart period variability and mortality after myocardial infarcperiod variability and mortality afte

4 Casolo G, Balli E, Taddei T, Amuhasi J, Gori C. Decreased spontaneous heart rate variability in congestive heart failure. Am $\mathcal{F}$ Cardiol 1989;64:1162-7. 